\title{
An updated list of Egyptian mosses
}

\author{
Wagieh El Saadawi \\ Afaf Badawi \\ Hanaa M. Shabbara \\ UsamaY. Abou Salama
}

and

Mahmoud S. M. Refai

Botany Department, Faculty of Science, Ain Shams University.

\section{Cairo-Egypt.}

E- mail: elsaadawy@link.com.eg

El-Saadawi W., Badawi A., Shabbara H.M., Abou Salama U.Y.\& Refai S.M., 1999. An updated list of Egyptian mosses. Taeckholmia 19(2): 77-96.

\begin{abstract}
An updated list provides information concerning the, hitherto, known Egyptian mosses (168 taxa). Reference is made to new records (20 taxa), phytogeographical territory(ies) from which each moss has been recorded, and other relevant data.

An annex to the main list includes the excluded taxa, which are 16 entities and are either nomina nuda, misidentified or, hitherto, recorded from only greenhouses and gardens or from beyond Egyptian boundaries. provided

An appendix including 67 reference works dealing entirely or partly with Egyptian mosses is also
\end{abstract}

Key words: Bryoflora, Egypt, Mosses.

\section{Introduction:}

Many works dealing entirely or partly with Egyptian mosses have appeared (see Appendix 1) since the publication of the basic list by El-Saadawi \& Badawi (1977) which then included 74 taxa. Records naturally increased, and the reported number, reached at present 424 taxa: 19 hepatics and 405 mosses. More than half the number of reported mosses, however, still requires careful investigation to reach precise identifications.

Earlier works on Egyptian mosses (all given in Appendix 1) included 16 taxa that should be excluded from the moss flora of Egypt, being misidentifications, nomina nuda, not collected from Egyptian territories or grow only in greenhouses and gardens. These 16 taxa are given at the end of this text.

The present account is an updated list of the Egyptian mosses, based on our present knowledge for the moss flora of Egypt. It is hoped to be a basic step towards a comprehensive future work. It includes 148 taxa from earlier publications together with 20 new records, i.e. a total of 168 entities. These 168 entities represent 139 species belonging to 44 genera, 14 families and 9 orders; all under class Bryopsida. All entities, except those marked with \#, are represented by specimens at CAIA and/or CAI.

Received 23 June 1999. Revision accepted 20 October 1999. 
Synonyms given in the present list are only those reported in the publications dealt with mosses from Egypt. The accepted names and the classification system used here are based mainly on Wijk et al. (1959-69); Crosby (1977, 1979), Corely et al. (1981), Crosby\& Bauer (1981, 1983), Anderson et al. (1990), Herrnstadt et al. (1991), Crosby et al. (1992), Zander (1993) and Crosby \& Magill (1994). Abbreviations of names of authors of plant names are as given in Brummitt \& Powell (1992). The presented list includes also the phytogeographical territory(ies) from which each moss has been recorded. These phytogeographical territories (Fig.1) are partially after Täckholm (1974) and El-Hadidi\& Fayed (1994/95). These territories are arranged according to date of publication for each record and are given in an abbreviated form. Explanations of these abbreviations are given in Figure 1, other abbreviations used in the list are as follows:

Eg: Egypt (unknown locality) including Sinai; E: Egypt (unknown locality) excluding Sinai; (R?): Species not certain; *: New record; \#: Specimens not at CAIA and/or CAI; $\checkmark$ : Collected mainly once before 1904; a: Not detected in any source of reference including indices.

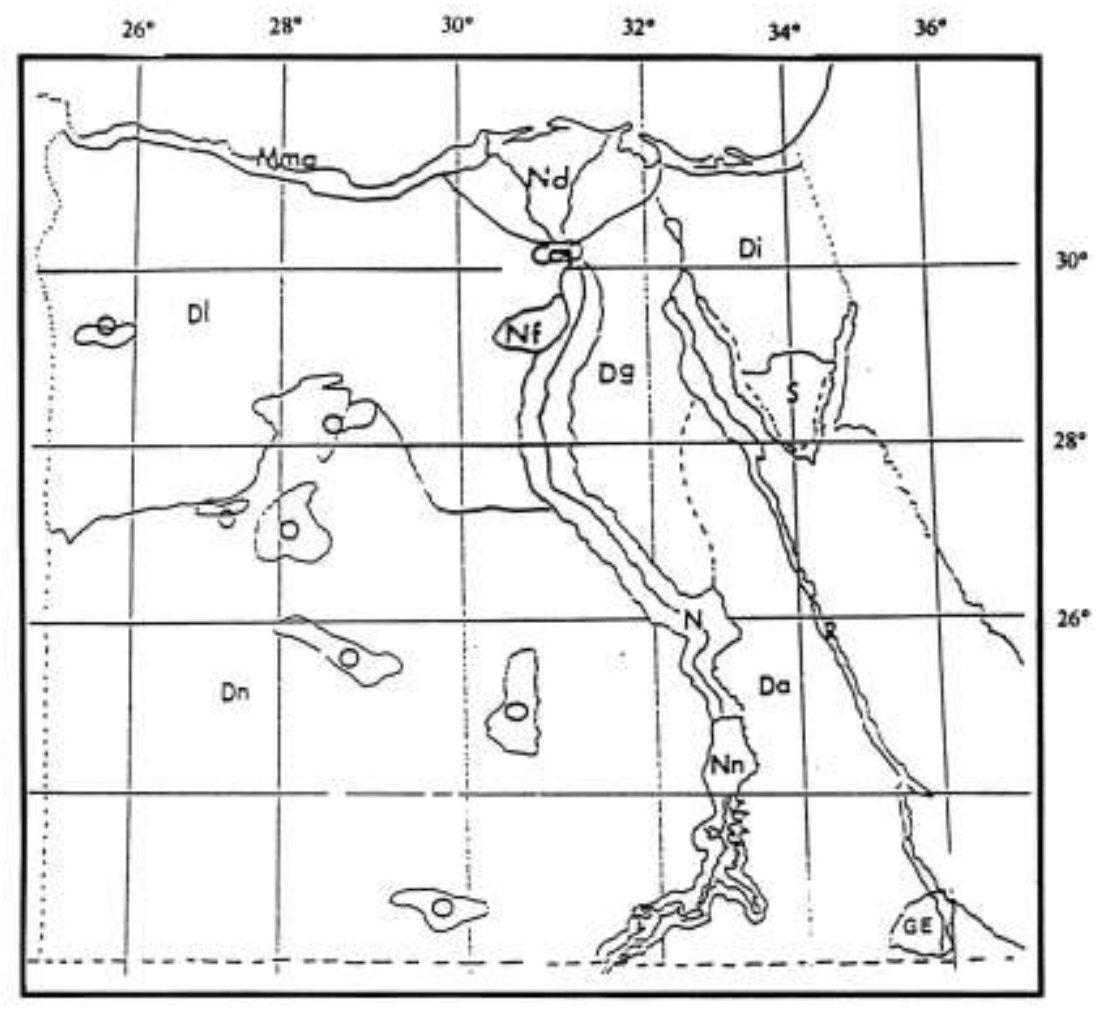

Fig.1. Phytogeographical territories in Egypt (After Täckholm, 1974; El- Hadidi \& Fayed 1994/95). Cai: Cairo area; Da: Arabian Desert; Dg: Galala Desert; Di: Isthmic Desert; DI: Libyan Desert; Dn: Nubian Desert; GE: Gebel Elba; Mma: Western Mediterranean coastal land; Nd: Nile Delta; Nf: Nile Faiyum; Nn: Nile Nubia, from Kom-Ombo southwards to Egyptian boundaries with the Sudan including the areas now inundated by the waters of Lake Naser since 1965; Nv: Nile Valley, from Cairo- Giza to Kom- Ombo; O: Oases of Western Desert; R: Red Sea costal plains; S: Southern Sinai massive (Sinai proper i.e. relatively high mountains, south of Isthmic desert). 


\section{CLASS: Bryopsida}

Order: Fissidentales

Family: Fissidentaceae Schimp.

1. Fissidens arnoldii R. Ruthe, Hedwigia 9: 178. 1870.

Syn. F.obtusifolius Wilson, 1845.

GE; S.

2. F. bryoides Hedw., Spec. Musc. 153. 1801.

Cai, Nd; Dg.

3. F. bryoides Hedw. var. gymnandrus (Büse) R. Ruthe, Hedwigia 9:178. 1870. Nf.

マ \# 4. F. crassipes Wilson ex Bruch, Schimp. \& W. Gümbel, Bryol. Eur. 1: 197. 100. 1849. Cai.

5. F. crassipes Wilson ex Bruch, Schimp. \& W. Gümbel ssp. warnstorfii (M. Fleisch.) Brugg.- Nann., Proceedings of the Koninklijke Nederlandsee Akademie van Wetenschappen, series C 85: 59-104. 1982

Syn. F. mnevidis J.J. Amann, 1922.; F. crassipes Wilson ex Bruch, Schimp. \& W. Gümbel var. philibertii Besch.,1882.

$\mathrm{E}, \mathrm{Eg} ; \mathrm{Nd}$.

6. F. limbatus Sull., Rep. Expl. Surv. Railr. R. Bot. 4 (5): 185.1. 1856.

Syn. F. alexandrinus Lorentz, 1867; F. cyprius Jur., 1865.

Mma, Nv (R?), Cai; S.

7. F. viridulus (Sw.) Wahlenb., Fl. Lapp. 334. 1812.

Syn. F.impar Mitt.,1885; F. bryoides Hedw. ssp. viridulus (Sw.) Kindb., 1897.

Cai, Nd, Nf, Dg, Mma, Nv, Nn; GE.

8. F. viridulus (Sw.) Wahlenb. var. bambergeri (Schimp.) Waldh. in Weim., Foert. Skand. Vaext. Moss2: 18. 1937.

Syn. F. viridulus ssp. bambergeri (Schimp.) Kindb., 1897.

Cai.

• \# 9. $\quad$ F. fontanus (Bach. Pyl.) Steud., Nom. Bot. 2: 166. 1824.

Syn. F. julianus (Sav.) Schimp., 1838.

E.

Order: Dicranales

Family: Ditrichaceae Limpr.

10. Ceratodon purpureus (Hedw.) Brid. var. rotundifolius Berggr., K. Svensk.Vet.

Ak. Handl. 13 (7): 44. 1875.

Syn. C. purpureus (Hedw.) Brid. var. obtusifolius Limpr., 1887.

Nf.

Family: Dicranaceae Schimp.

$\leftarrow$ Subfamily: Anisothecioideae

11. Dicranella rufescens (With.) Schimp.,Coroll. 13.1856.

Syn. Anisothecium rufescens (With.) Lindb. 1879.

Nf.

$\uparrow$ Subfamily: Cynodontoideae

12. Dichodontium pellucidum (Hedw.) Schimp., Coroll. 12. 1856.

Syn. D. pellucidum (Hedw.) Schimp. var. fagimontanum (Brid.) Schimp., 1856;

D. pellucidum (Hedw.) Schimp. fo. fagimontanum C.E.O. Jensen, 1939.

Cai.

Order: Encalyptales

Family: Encalyptaceae Schimp. 
W. El-Saadawi, A. Badawi, H.M. Shabbara, U.Y. Abou Salama \& M.S.M. Refai

13. Encalypta intermedia Jur., Verh. Zool. Bot. Ges. Wien 20: 595. 1870.

Syn. E. vulgaris Hedw. var. mediolanensis Warnst., 1899.

$\mathrm{S}$.

\# 14. E. vulgaris Hedw., Spec. Musc. 60.1801.

S.

Order: Pottiales

Family: Pottiiaceae Schimp.

$\leftarrow$ Subfamily: Timmielloideae

15. Timmiella barbuloides (Brid.) Mönk., Laubm. Eur. 273. 1927.

Syn. T. barbula (Schwägr.) Limpr., 1888.

[ As T. anomala by Abou Salama 1985: 99]

GE; S.

$\uparrow$ Subfamily: Trichostomoideae

16. Eucladium verticillatum (Brid.) Bruch, Schimp. \& W. Gümbel, Bryol. Eur. 1: 9. 40. 1846.

Syn. E. verticillatum forma [Lorentz 1867: 15]; Hymenostylium verticillatum [Hart 1891: 169]; Eucladium verticillatum (L.) Bryol. eur.[Brotherus 1924:

258].

$\mathrm{S}$, Cai; R.

17. Tortella inflexa (Bruch) Broth., Nat. Pfl. 1 (3): 397. 1902.

Mma.

18. T. nitida (Lindb.) Broth., Nat. Pfl. 1 (3): 397. 1902.

Syn. Barbula alexandrina Lorentz, 1867; Barbula muralis var. alexandrina Müll. Hal. [Sickenberger 1901:317]; Barbula nitida Lindb. [Sickenberger 1901: 317] Mma,GE; Nv.

* 19. T. tortuosa (Hedw.) Limpr., Laubm. 1: 604. 1888.

Dg.

* 20. Trichostomum crispulum Bruch in F.A.Müll., Flora 12: 395. 4. 1829.

$\mathrm{S}$.

$\rightarrow$ Subfamily: Merceyoideae

21. Barbula convoluta Hedw., Spec. Musc. 120. 1801.

Syn. Streblotrichum convolutum (Hedw.) P. Beauv., 1805.

$\mathrm{Nn}, \mathrm{Dg} ; \mathrm{S}$.

22. B. ehrenbergii (Lorentz) M. Fleisch., Musci Arch. Indic. ser. 4:n. 161.1901.

Syn. Trichostomum ehrenbergii Lorentz, 1867; Didymodon ehrenbergii (Lorentz) Kindb.,1897; Hydrogonium ehrenbergii (Lorentz) A. Jaeger, 1880; Barbula bolleana (Müll.Hal.) Broth., 1924 [cf. Ros et al. 1999].

S, Cai, Nd, Nf, Nv; Nn.

23. B. indica (Hook.) Spreng. in Steud., Nomencl. Bot. 2: 72.1824

Syn. Semibarbula orientalis (F. Weber) Wijk \& Margad., 1959.

Cai, Nd; Nv.

24. B. unguiculata Hedw., Spec. Musc. 118.1801.

Cai, Nd, Nf, Dg; Nv.

25. B. unguiculata Hedw. fo. cuspidata (Schultz) Mönk., Laubm. Eur.286. 1927.

Syn. B. unguiculata Hedw. var. cuspidata (Schultz) Brid. 1826.

Nf; Dg

26. B. unguiculata Hedw. fo. obtusifolia Mönk., Laubm. Eur. 286.1927.

Cai; Nd. 
27. B. unguiculata Hedw. fo. robusta (Lindb.) Podp., Consp. 214. 1954.

Syn. B. unguiculata Hedw. var. robusta Lindb. 1929.

Nf.

28. Didymodon aaronis (Lorentz) J. Guerra in J. Guerra \& Ros, Cryptogamie Bryol. Lichénol. 8: 55. 1987.

Syn. Trichostomum aaronis Lorentz, 1867; Barbula aaronis (Lorentz) Hilp., 1933; Gyroweisia aaronis (Lorentz) Par. [cf. Hume 1906: 229]; Trichostomopsis aaronis (Lorentz) S. Agnew \& Tonwnsend, 1970.

[As Barbula sp.4 by Abou Salama 1985: 93]

S; Dg.

29. D. fallax (Hedw.) R.H. Zander, Phytologia, 41: 28. 1978.

[As Barbula acuta by Abou Salama 1985: 87, as Didymodon acutus by Shabbara 1986: 52; Badawi et al.1987b: 359; El- Saadawi et al.1987b: 334.]

$\mathrm{S} ; \mathrm{Nf}$.

* 30. D. fallax (Hedw.) R.H. Zander var. reflexus (Brid.) R.H. Zander, Bryologist 83:230. 1980.

Syn. Barbula reflexa (Brid.) Brid., 1819

Mma.

31. D. luridus Hornsch. in Spreng. Syst. Veg. 4 (1): 173.1827.

Syn. D. trifarius (Hedw.) Röhl., 1813.

$\mathrm{S}, \mathrm{Mma} ; \mathrm{Dg}$.

* 32. D. rigidulus Hedw. ,Spec. Musc.104.1801.

Syn. Barbula bescherellei Sauerb.,1880

S.

33. D. rigidulus Hedw. var. gracilis (Schleich. ex Hook. \& Grev.) R.H. Zander, Cryptogamie Bryol. Lichénol. 2 : 393. 1981.

Syn. B. gracilis (Schleich.) Schwägr. var. viridis Br. eur.[Geheeb 1903: 187]; Barbula acuta (Brid.) Brid.,1819; Didymodon acutus (Brid.) K. Saito.,1975; Didymodon acutus (Brid.) K. Saito f. viridis (Bruch, Schimp. \& W. Gümbel) Podp. [Vana 1988].

$\mathrm{S}, \mathrm{Mma}$; Nf.

34. D. sinuosus (Mitt.) Delogne. Bull. Soc. Roy. Bot. Belg., 12: 423. 1873.

Syn. Barbula sinuosa (Mitt.) Grav., 1874.

Nf; Mma.

* 35. D. spadiceus (Mitt.)Limpr., Laubm. Deutschl. 1:556. 1888.

$\mathrm{S} ; \mathrm{Dg}$.

\# 36. D. tectorum (Müll. Hal.) K. Saito.,J. Hattori Bot. Lab. 39:516. 1975.

Syn. Barbula tectorum Müll. Hal., 1896. Mma.

37. D. tophaceus (Brid.) Lisa, Elenco. Muschi Torino, 31. 1837.

Syn. Trichostomum tophaceum forma foliis magis acuminatis [Lorentz 1867:21] ; Barbula tophacea (Brid.) Mitt.,1859.

$\mathrm{S}$, Cai, Nd, Mma, Dg, Nf, Nv; Nn.

* 38. D. tophaceus (Brid.) Lisa var. brevifolius (Bruch, Schimp. \& W. Gümbel) Paris, Ind. Bryol.ed. 2,2: 77.1904.

$\mathrm{S}$.

\# 39. D. tophaceus (Brid.) Lisa var. humilis (Schimp.) Warnst. Krypt. Fl. Brandenburg 2: 230.1904

S; GE. 
W. El-Saadawi, A. Badawi, H.M. Shabbara, U.Y. Abou Salama \& M.S.M. Refai

\# 40. D. tophaceus (Brid.) Lisa fo. lingulatus (Boulay) Mönk., Süsswasserfl. 14:67.1914. Syn. Barbula tophacea fo. lingulata.[El- Saadawi \& Badawi 1977: 182] Eg.

41. D. vinealis (Brid.) R.H. Zander, Phytologia 41: 25. 1978.

Syn. Barbula vinealis Brid., 1827; Barbula cylinderica (Taylor) Schimp., 1872;

Barbula vinealis (Brid.) ssp. cylindrica (Taylor) Podp. ,1954.

[As Barbula 1 by Shabbara 1986: 51; Badawi et al. 1987b: 359; El Saadawi et al. 1987b: 334]

S, Mma, Nd, Cai, Nf; Di.

42. Gymnostomum aeruginosum Sm., Fl. Brit. 3: 1163. 1804.

Syn. Hymenostylium rupestre Schwägr. [Hart 1891: 169]

[As Hymenostylium recurvirostre by Abou Salama 1991: 85]

S, Nf; Cai.

43. G. calcareum Nees \& Hornsch., Bryol. Germ. 1: 153. 10 f. 15.1823.

Nv, Dg, GE, S, Nd, Nf; Mma.

\# 44. G. calcareum Nees \& Hornsch. var. muticum Boulay, Musci France 556. 1884. $\mathrm{S}$.

45. G. mosis (Lorentz) Jur. \& Milde, Verh. Zool. Bor. Ges. Wien. 20: 590. 1870. Syn. Trichostomum mosis Lorentz, 1867; Gyroweisia mosis (Lorentz) Paris 1896 [As Gymnostomum sp. 5 by Abou Salama 1991: 92] S.

46. G. viridulum Brid. fid. Whitehouse \& Crundw., J. Bryol. 16: 563. 1991.

Syn. Weissia rohlfsiana Müll. Hal.,1874; Gymnostomum calcareum Nees \&

Hornsch. var. viridulum ( Brid.) Br. eur., 1846; Gyroweisia rohlfsiana (Müll. Hal.) Paris, 1896.

[As? Gymnostomiella sp. by Abou Salama 1985: 114, as Gymnostomiella orcuttii by Shabbara 1990: 179]

Nv, Cai, Mma, Dg; S.

47. Gyroweisia reflexa (Brid.) Schimp., Syn. ed. 2: 39. 1876.

[As G. tenuis by Yousef 1987: 20; by Lashin 1990:32]

Cai, S, Nd; Dg.

48. G. tenuis (Hedw.) Schimp., Syn. ed. 2: 38.1876.

$\mathrm{S}$.

49. Hymenostylium recurvirostrum (Hedw.) Dixon, Rev. Bryol. Lich.6: 96. 1934.

Syn. Gymnostomum recurvirostre Hedw., 1801.

[ As Moss 2 by Abou Salama 1985: 128]

S.

50. Pseudocrossidium hornschuchianum (Schultz) R.H. Zander, Phytologia 44: 205. 1979.

Syn. Barbula hornschuchiana Schultz, 1822.

Mma, S; Nv.

$\downarrow$ Subfamily: Pottioideae

51. Aloina aloides (Schultz) Kindb., Bih. K. Svensk. Vet. Ak. Handl. 7 (9): 136.1883.

Syn. Barbula aloides [Lorentz 1867:31]

$\mathrm{S} ;$ Mma. 
52. A. aloides (Schultz) Kindb. var. ambigua (Bruch, Schimp. \& W. Gümbel) E.J. Craig in Grout, Moss Fl. N. Am. 1(4):214. 1939.

Syn. A. ambigua (Bruch \& Schimp.) Limpr.,1888; A. rigida (Hedw.) Limpr. var. ambigua (Bruch, Schimp. \& W. Gümbel) E.J. Craig.,1940.

Mma; S.

53. A. bifrons (De Not.) Delgad., Bryologist 76: 273. 1973.

Syn. A. rigida (Hedw.) Limpr. var. pilifera (De Not.) Limpr.,1888; A. pilifera (De Not.) H.A. Crum \& Steere, 1959.

Mma.

"*"54. A. rigida (Hedw.) Limpr., Laubm. Deutschl. 1: 637.1888.

Mma.

55. Crossidium aberrans Holz. \& E.B. Bartram, Bryologist 27: 4. 2f.1.1924.

[As C. chloronotos by Abou Salama 1985: 101, as C. crassinerve by Abou Salama 1991: 107]

S, Mma; Di.

56. C. crassinerve (De Not.) Jur., Laubmoosfl. Oest. Ungarn 128.1882.

[As C. chloronotos (Brid.) Limpr., 1888 by Imam \& Ghabbour 1972: 520; cf. Ros et al. 1999].

Mma.

\# 57. C. geheebii (Broth.) Broth., Nat. Pfl. Fam. 1(3): 426.1902

[As C. aberrans by Delgadillo 1975: 271; cf. Frey \& Kürschner 1988 a,b] S.

* 58. C. laevipilum Thér. \& Trab., Bull. Soc. Hist. Nat. Afr. Nord., 22:161. 3. 1931 Mma.

\# 59. C. squamiferum (Viv.) Jur., Laubmoosfl. Oest. Ungarn. 127. 1882.

Syn. Barbula membranifolia [Lorentz 1867:31]; Barbula membranifolia Hook. [Sickenberger 1901: 317].

S; Di.

* 60. C. squamiferum (Viv.) Jur. var. pottioideum (De Not.) Mönk., Laubm. Eur. 315. 1927.

$\mathrm{S}$.

* 61. Hennediella stanfordensis (Steere) Blockeel, J. Bryol. 16: 191, 1991. Syn. Hyophila stanfordensis (Steere) Smith \& Whitehouse, 1974. Nd.

62. Microbryum starckeanum (Hedw.) R.H. Zander, Bull. Buffalo. Soc. Nat. Sc. 32: 313.1993

Syn. Pottia starckeana (Hedw.) Müll. Hal.,1849.

Mma; S.

63. M. starckeanum (Hedw.) R.H. Zander var. brachyodus (Bruch, Schimp. \& W. Gümbel) R.H. Zander, Bull. Buffalo. Soc. Nat. Sc. 32: 313. 1993.

Syn. Pottia mutica Venturi, 1869; Pottia starckeana agg. (Hedw.) Müll Hal. [cf. Ros et al., 1999]

Cai; S.

\# 64. Pterygoneurum subsessile (Brid.) Jur., Laubmoosfl. Oest. Ungarn 96. 1882.

Di.

\footnotetext{
"**" Not a new record any more, see Gallego et al. 1999.
} 
W. El-Saadawi, A. Badawi, H.M. Shabbara, U.Y. Abou Salama \& M.S.M. Refai

65. Syntrichia inermis (Brid.) Bruch in Huebener, Musc. Germ. 335. 1833.

Syn. Barbula inermis [Lorentz 1867: 32] ;Tortula inermis Mont., [ Hart 1891: 169 ]

$$
\text { S. }
$$

• \# 66. S. rigescens (Broth. \& Geh. in Broth.) Ochyra, Fragm. Florist. Geobot. 37: 212. 1992.

Syn. Tortula rigescens Broth. \& Geh.,1909.

S.

67. Tortula atrovirens (Sm.) Lindb., Oefv. K.Vet. Ak. Foerh. 21 (4): 236.1864. Syn. Desmatodon convolutus (Brid.) Grout,1939.

S; Mma.

*68. T. brevissima Schiffn., Ann. Naturh. Hofmus. Wien 27:481.23-33. 1913. $\mathrm{S}$.

\# 69. T. canescens Mont., Arch. Bot. 1 (2): 133. 4f. 3. 1833.

$$
\text { S. }
$$

\# 70. T. kneuckeri Broth. \& Geh., Nat. Pfl. Fam. ed. 2, 10: 297.1924.

$\mathrm{S}$.

* 71. T. marginata (Bruch \& Schimp.) Spruce, London J. Bot. 4: 192. 1845. Mma.

* 72. T. modica R.H. Zander, Bull. Buffalo. Soc. Nat. Sc. 32: 333.1993. Syn. Pottia intermedia (Turner) Fürnr., 1829. Mma.

73. T. muralis Hedw., Spec. Musc. 123. 1801.

[ As T. muralis Hedw. var. aestiva Brid. ex Hedw. by Shabbara 1990: 157] Cai, Mma, Nd; Dg.

74. T. muralis Hedw. fo. incana (Bruch, Schimp. \& W. Gümbel) Sapjegin, Bot. Jahrb. System. 46 (Beibl.105) :14, 1911.

Syn. Barbula muralis var. incana Bruch, Schimp. \& W. Gümbel, 1842; Tortula muralis Hedw. var. incana Br. eur. [Imam \& Ghabbour 1972: 522] Mma.

75. T. muralis Hedw. var. obcordata (Schimp.) Limpr. Laubm. Deutschl. 1: 666. 1888. Syn. T. muralis Hedw. fo. obcordata (Schimp.) Mönk., 1927. Mma.

76. T. pallida (Lindb.) R.H. Zander var. longicuspis (Warnst.) R.H. Zander, Bull. Buffalo. Soc. Nat. Sc. 32: 319.1993.

Syn. Pottia venusta Jur. [Lorentz 1867: 21]; Pottia pallida Lindb. var. longicuspis Warnst., 1916; Pottia pallida Lindb., 1864 [ cf. Ros et al. 1999] Mma

77. T. plinthobia (Sull. \& Lesq.) Austin, Musci Appalach. Suppl. 1:10. 1878. cf. R.H. Zander, Bryologist 82: 551. 1979.

Syn. Desmatodon plinthobius Sull. \& Lesq., 1856. Mma.

78. T. vahliana (Schultz) Mont., in Gay, Hist, Fis. Polit. Chil. Bot. 7:153.1850. Mma.

79. T. wilsonii (Hook.) R.H. Zander, Bull. Buffalo. Soc. Nat. Sc. 32: 320.1993. Syn. Pottia wilsonii (Hook.) Bruch, Schimp. \& W. Gümbel , 1843. Mma.

* 80. Weissia controversa Hedw., Spec. Musc.67.1801. $\mathrm{S}$. 
• \# 81. W. longifolia Mitt. fid. Crundw. \& Nyholm, J. Bryol. 7: 13. 1972.

Syn. Systegium crispum (Hedw.) [Lorentz 1867: 15]; Astomum crispum(Hedw.) Hampe, 1837.

Mma.

Family: Cinclidotaceae (Broth.) Mönk.

\# 82. Cinclidotus mucronatus (Brid.) Guim., Cat. Descr. Briol. Portug. 57. 1919. $\mathrm{S}$.

\# * 83. C. riparius (Brid.) Arn., Mem. Soc. Linn. Paris 7 : 247. 1827.

$\mathrm{S}$.

Order : Grimmiales

Family: Grimmiaceae Arnold

84. Grimmia anodon Bruch \& Schimp. , Bryol. Eur. 3: 110. 236. 1845.

S.

\ \# 85. G. anodon Bruch \& Schimp. var. sinaitica Renauld \& Cardot, Bull. Herb. Boiss. 2:33. 1894.

S.

86. G. crinita Brid., Spec. Musc. 1: 95.1806

Syn. G. sinaica Hampe. [Lorentz 1867: 35; Geheeb 1903: 204]

S.

• \# 87. G. laevigata (Brid.) Brid., Bryol. Univ., 1: 183.1826.

Syn. G. campestris Burch ex Hook. 1819; G. leucophaea Grev. [Hart 1891: 169] $\mathrm{S}$.

\# 88. G. orbicularis Bruch in Wilson, Engl. Bot. Suppl. 4: n. 288. 1844.

$\mathrm{S}$.

• \# 89. Racomitrium aciculare (Hedw.) Brid., Mant. Musc. 80 .1819.

Syn. Trichostomum aciculare P. Beauv. [Decaisne 1834 cf. Hart 1891: 171]

S.

90. Schistidium agassizii Sull. \& Lesq., Musci Bor. Am. 31. 1856.

Syn. Grimmia alpicola Sw. [Geheeb 1903: 203]; Schistidium alpicola (Hedw.) Limpr., 1889.

[As Grimmia (Schistidium) alpicola Sw. fo. sinaitica by Geheeb 1903: 203]. $\mathrm{S}$.

• \# 91. S. apocarpum (Hedw.) Bruch \& Schimp., Bryol. Eur. 3: 99. 233. 1845.

Syn. Grimmia apocarpa L. [Hart 1891: 168]

$\mathrm{S}$.

Order: Funariales

OSuborder: Funariineae

Family: Funariaceae Schwägr.

• \# 92. Entosthodon attenuatus (Dicks.) Bryhn, K.Norsk. Vid. Selsk. Skrift. 1908 (8): 25. 1908.

Syn. E. templetonii Schwägr. [Schimp. 1876: 380; Hart 1891: 170]; Funaria attenuata Dicks.) Lindb., 1870.

$\mathrm{S} ; \mathrm{E}$.

• \# 93. E. curvi-apiculatus Müll. Hal., Flora 57: 483. 1874.

Syn. Funaria curvi-apiculata (Müll.Hal.) Broth.,1903.

Nv. 
W. El-Saadawi, A. Badawi, H.M. Shabbara, U.Y. Abou Salama \& M.S.M. Refai

94. E. durieui Mont., Ann. Sc. Nat. Bot. Ser. 3, 11:33. 1849.

Syn. E. pallescens Jur. fo. foliis solidioribus [Lorentz 1867:38]; Funaria pallescens ( Jur.) Lindb., 1888., Entosthodon pallescens Jur., 1865

Cai, GE; S.

\# 95. E. fascicularis (Hedw.) Müll. Hal., Syn. 1: 120. 1848.

Syn . Funaria fascicularis (Hedw.) Lindb.,1865.

Cai.

\# 96. E. niloticus Schimp., Bot. Zeit. 13: 747. 1855.

Syn. F. nilotica Broth., 1903. [Entosthodon niloticus (Delile) Schimp. = Physcomitrium niloticum (Delile) Müll. Hal. et pp.non typ. Entosthodon niloticus Schimp.]

Cai; Dg

\# 97. E. obtusus (Hedw.) Lindb., Oefv. K. Vid. Ak. Foerh. 21: 221. 1865.

Syn. Funaria obtusa (Hedw.) Lindb.,1870.

Cai.

98. E. plano-convexus (E.B. Bartram) Grout, Moss. Fl. N. Am. 2: 80. 34.1935. Di.

• \# 99. Funaria anomala Jur. in Ung. \& Kotschy, Ins. Cypern 170. 1865.

Cai.

* 100. F. convexa Spruce, Musc. Pyren. no. 149. 1847.

[As F. calcarea var. mediterranea by Abou Salama 1985:111; Abou Salama 1991: 126]

S.

\# 101. F. handelii Schiffn., Ann. Naturh. Hofmus Wien 27: 493. 61- 69. 1913. Cai; Dg.

102. F. hygrometrica Hedw., Spec. Musc. 172.1801.

Cai, Nv, Nn, Nd, Dg, S, Nf, Mma; Di.

103. F. hygrometrica Hedw. var. calvescens (Schwägr.) Mont., Ann. Sc. Nat. Bot. ser. 2,12: 54.1839 .

Di.

104. F. hygrometrica Hedw. var. intermedia Warnst., Verh. Bot. Ver. Branden. 42: 200. 1901.

Dg.

\# 105. F. hygrometrica Hedw. var. minor (Delile) Brid., Bryol. Univ. 2: 738. 1827. Syn. F. minor Delile, 1813.

Cai.

\# 106. F. hygrometrica Hedw. var. muralis Huebener, Musc. Germ. 497.1833.

Syn. F. hygrometrica Hedw. var. patula Br. eur., 1846 [Imam \& Ghabbour 1972: 521].

Dg.

• \# 107. F. microstoma Bruch ex Schimp., Flora 23: 850.1840.

Eg.

108. F. muhlenbergii Turner, Ann. Bot. 2 (1): 198, 1805.

Syn. Funaria dentata Crom. var. mediterranea ( Lindb.) Limpr., 1891; F. calcarea Wahlenb. var. mediterranea (Lindb.) C. E. O. Jensen \& Medelius, 1929; F. mediterranea Lindb. 1863.

E, GE; Cai.

\# 109. F. nubica Müll. Hal., Hedwigia 38: 61. 1899.

$\mathrm{Nn}$. 
110. F. pulchella H. Philib., Rev. Bryol. 11: 41. 1884.

E; GE "new record to GE".

— 111. F. sickenbergeri Müll. Hal., Gen. Musc. Fr. 105. 1900.

O.

112. Micropoma niloticum (Delile) Lindb. in Broth., Nat. Pfl.1 (3): 518. 372. 1903. Syn. Gymnostomum niloticum Delile,1813; Physcomitrium niloticum Lindb. [Müll. Hal.,1858: 154]; Microstegium niloticum (Delile) Lindb., 1865; Physcomitrium sesostris Lorentz, 1867; Physcomitrium niloticum (Delile) Brid. [Sickenberger 1901: 316].

Cai, Nv, Nd, GE, Nf; Dg.

\# 113. Physcomitrium eurystomum Sendtn. Denkschr. Bayer. Bot. Ges. Regensburg 3: 142.1841

Syn. P. acuminatum (Schleich.) Bruch, Schimp. \& W. Gümbel, 1841;

Physcomitrium eurystomum Sendtn. fo. acuminatum (Bruch, Schimp. \& W.

Gümbel) Löske, 1929.

Cai; Dg.

114. P. immersum Sull., in Gray, Man. Bot. N. U. States 648. 1848.

Di.

115. P. pyriforme (Hedw.) Brid., Bryol. Univ. 2: 815. 1827.

Cai, Nn, Nd.; Dg.

-Suborder : Splachineae

Family: Splachnobryaceae A. Kop.

* 116. Splachnobryum limbatum D.H. Norris \& R.H. Zander, The Bryologist 84 (3): 404. 1981.

Mma.

* 117. S. obtusum (Brid.) Müll. Hal., Verh. Zool. Bot. Ges. Wien 19: 504. 1869.

[As Philonotis gracillima by Shabbara, 1986: 71; Badawi et al. 1987b: 359; ElSaadawi et al. [1987b: 349]

Nf.

Order: Bryales

Family: Bryaceae Schwägr.

118. Brachymenium cf. angustirete Ochi, Fac. of Education, Tottori Univ., 23 (1): 24. 1972.

$\mathrm{Nd}$.

\# 119. B exile (Dozy. \& Molk.) Bosch \& Sande Lac., Bryol. Jav. 1: 139. 1860.

[As Bryum (?) comense, cf. Ochi 1972: 8.; as Bryum caespiticium L. by Geheeb 1904: 4, rev. Ochi cf. Vana 1988.]

$\mathrm{S} ;$ Mma.

120. B. cf. longicolle Thér., Bull. Soc. Bot. Geneve, 26: 85. ic. 1936.

$\mathrm{Nd}$.

121. B. cf. sikkimense Renauld \& Cardot, Bull. Soc. R. Bot. Belg. 38 (1): 12.1900. $\mathrm{S}$.

122. Bryum alpinum Huds. ex With., Syst. Arr. Brit. Pl. ed. 4,3: 824. 1801.

[As B. alpinum ssp. gemmiparum by El- Awadi 1985: 64; El- Saadawi et al. 1987a: 301].

Cai, Nf; S.

\# 123. B. alpinum Huds. ex With. var. viride Husn., Musc. Gall. 297.67 f. 6. 1889. Nn. 
124. B. cf. apiculatum Schwägr., Spec. Musc. Suppl. 1 (2): 102. 72.1816. $\mathrm{Nd}$.

125. B. argenteum Hedw. Spec. Musc. 181. 1801.

Cai, Mma; S.

126. B. argenteum Hedw. var. lanatum (P. Beauv.) Hampe 1839.

Dg; S.

127. B. bicolor Dicks., Pl. Crypt. Brit. fasc. 4: 16. 1801.

Syn. Bryum dichotomum Hedw. 1801.

S, Cai, Nv, Mma, Dg, Nd; Nf.

128. B. caespiticium Hedw., Spec. Musc. 180. 1801.

Cai, Mma, S; Nd.

129. B. caespiticium Hedw. var. badium Brid., Bryol. Univ. 1: 850. 1827. Syn. B. badium (Brid.) Schimp., 1876.

Cai, Dg, Mma; S.

130. B. capillare Hedw., Spec. Musc. 182. 1801.

Syn. B. aschersonii Müll. Hal. 1874.

[As B. syriacum Lorentz, cf. Ochi 1972: 91].

$\mathrm{O}, \mathrm{S}, \mathrm{Cai}$; Dg.

\# 131. B. cellulare Hook. in Schwägr., Spec. Musc. Suppl. 3 (1): 214a. 1827.

Cai; Dg.

132. B. comense Schimp. Syn. ed. 2: 444. 1876.

Syn. B. caespiticium Hedw. var. comense (Schimp.) Husn., 1889; B. caespiticium Hedw. ssp. comense (Schimp.) J.J. Amann, 1893.

Mma, Cai; Nd.

* 133. B. creberrimum Taylor, London J. Bot. 5: 54. 1846.

S.

134. B. elegans Nees in Brid., Bryol. Univ. 1: 849. 1827.

Syn. B. capillare Hedw. ssp. elegans (Nees) Lindb., 1879.

[As B. capillare by Abou Salama 1985: 117].

S; Nf.

135. B. funkii Schwägr., Spec. Musc. 1(2): 89. 69. 1816.

Dg; $\mathrm{S}$.

136. B. cf. gedeanum Bosch \& Sande Lac., Bryol. Jav. 1: 147. 120. 1860. $\mathrm{Nd}$.

137. B. gemmiparum De Not., Cronac. Briol. Ital. 1: 26. 1866.

Syn. B. gemmiparum De Not. var. sinaica Geh., 1904; B. alpinum ssp. gemmiparum f. sinaica Podp.1953; B. alpinum With var. gemmiparum (De Not.) Lindb; B. alpinum Huds. ex With ssp. gemmiparum (De Not.) Kindb. 1897.

S, Cai ; Nf.

138. B. kunzei Hornsch., Flora 2 ( 1 ): 90. 1819.

Syn. B. caespiticium Hedw. var. imbricatum Bruch, Schimp. \& W. Gümbel, 1839.

Cai; Mma.

* 139. B. pseudotriquetrum (Hedw.) P. Gaertn., G. Mey. \& Scherb., Oek. Techn. Fl. Wetterau 3: 102. 1802.

[As B. syriacum by Geheeb 1904: 4, rev.Ochi, cf. Vana 1988] $\mathrm{S}$. 
* 140. B. pseudotriquetrum (Hedw.) P. Gaertn., G. Mey. \& Scherb. var. bimum (Brid.) Lilj., Utkast Svensk. Fl. ed. 3: 553. 1816.

[As Bryum sp. 6 by Abou Salama 1985: 124]

S.

141. B. radiculosum Brid., Spec. Musc. 3: 18.1817.

Syn. B. murorum (Schimp.) Berk., 1863.

Cai, Mma, S; Nd.

• \# 142. B. remelei Müll. Hal., Flora 57: 484. 1874.

Nv.

\# 143. B. sacrum (Lorentz) Müll. Hal., Gen. Musc. Fr. 222.1900.

Syn. Webera sacra Lorentz, 1868. [This may be B. syriacum cf. Geheeb 1904:4]. S.

\# 144. B. schleicheri Lam. \& Dc.[cf. Ros et al. 1999].

S.

145. B. syriacum Lorentz, Abhandl. Ak. Wiss. Berlin 1867: 45. 2. 1868.

Syn. Webera sinaitica Lorentz, 1868.

$\mathrm{S}$.

\# 146. B. syriacum Lorentz var. humile Schiffn., Ann. Naturh. Hofmus. Wien 27: 497. 1913.

Mma.

\# 147. B. torquescense Bruch ex De Not., Syll. N. 163. 1838.

Syn. B. capillare Hedw. ssp. torquescens (De Not.) Kindb., 1897.

Dg.

148. B. turbinatum (Hedw.) Turner, Musc. Hib. 127. 1804.

Syn. B. turbinatum Sw. [Decaisne 1834, cf. Hart 1891: 171]; B. turbinatum Hedw.[Hart 1891:170]

[As B. syriacum by Geheeb 1904: 4, rev. Ochi, cf. Vana 1988; as B.sp.4 by Abou Salama 1985: 122].

$\mathrm{S}$.

149. Leptobryum pyriforme (Hedw.) Wilson, Bryol. Brit. 219. 1855.

Cai, Nd; Mma.

\# 150. Pohlia atropurpurea (Wahlenb.) Lindb., Acta Soc. F. Fl. Fenn. 16: 14. 1899.

Syn. Bryum atropurpureum Wahlenb., 1829.

S.

• \# 151. P. korbiana (Müll. Hal.) Wijk \& Margad., Taxon 8: 74. 1959.

Syn. Bryum korbianum Müll. Hal., 1874.

O.

\# 152. P. melanodon (Brid.) A.J. Shaw. [cf. Ros et al. 1999].

Syn. Webera carnea [Lorentz 1867: 45] ; Pohlia carnea (Schimp.) Lindb., 1879; Mniobryum carneum ( L.) Limpr. [Brotherus 1924: 363]; Mniobryum delicatulum (Hedw.) Dixon, 1934.

Mma.

153. P. nutans (Hedw.) Lindb., Musci Scand. 18. 1879.

Cai; Mma.

Family: Bartramiaceae Schwägr.

154. Philonotis evanidinervis M. Fleisch., Musci Fl. Buitenzorg 2: 612. 116. 1904. $\mathrm{Nf}, \mathrm{Nd}$; Dg. 
\# 155. P. fontana ( Hedw.) Brid., Bryol. Univ.2: 18. 1827.

Syn. Philonotula glabriuscula Müll. Hal. [Sickenberger 1901:317]. [Philonotis glabriuscula kindb. In Macoun = pp. Philonotis fontana (Hedw.) Brid. et pp. Philonotis marchica (Hedw.) Brid.]

Cai.

156. P. hastata (Duby) Wijk \& Margad., Taxon 8: 74. 1959.

Syn. P. obtusata Müll. Hal. ex Renauld \& Cardot 1896.

Cai, Nd, Nf, O; Dg.

157. P. marchica (Hedw.) Brid., Bryol. Univ. 2: 23. 735. 1827.

Syn. Philonotula glabriuscula Müll. Hal.[ Sickenberger 1901: 317]

Cai; Nf.

158. P. marchica (Hedw.) Brid. fo. rivularis (Warnst.) Mönk., Laubm. Eur. 582. 1927. Cai.

Order: Orthotrichales

Family: Orthotrichaceae Arnold

159. Zygodon obtusifolius Hook., Musci Exot. 2: 159. 1819.

Nf.

Order: Hypnales

Family: Amblystegiaceae G. Roth

160. Amblystegium riparium (Hedw.) Bruch, Schimp.\& W. Gümbel Bryol. Eur. 6: 58. 570. 1853.

Syn. Leptodictyum riparium (Hedw.) Warnst., 1906.

Cai; S.

\# 161. Drepanocladus aduncus (Hedw.) Warnst., Beih. Bot. Centralbl. 13:400. 1903.

Syn. Hypnum aduncum L. [Decaisne 1834, cf.Hart 1891: 171.]

$\mathrm{S}$.

Family: Brachytheciaceae Schimp.

162. Brachythecium rivulare Bruch, Schimp.\& W. Gümbel Bryol. Eur. 6: 17. 546. 1853.

Cai; Nd.

\# 163. B. umbilicatum Jur. \& Milde, Verh. Zool. Bot. Ges. Wien 20: 600. 1870.

S.

\# 164. B. velutinum (Hedw.) Bruch, Schimp.\& W. Gümbel Bryol. Eur. 6: 9. 538. 1853.

Syn. Hypnum velutinum L. [Hart 1891: 170]

$\mathrm{S}$.

\# 165. B. velutinum ( Hedw.) Bruch, Schimp.\& W. Gümbel var. venustum ( De Not.) Arcang. Nuov. Giorn. Bot. Ital. 21: 471.1889.

Syn. B. venustum (De Not.) De Not., 1867.

$\mathrm{S}$.

\# 166. Eurhynchium pumilum (Wilson) Schimp., Coroll. 119. 1856.

Syn. Amblystegium burnatii J.J. Amann.,1895.

Nv; Cai.

\# 167. Rhynchostegiella tenella (Dicks.) Limpr., Laubm. Deutschl. 3: 209. 1890.

Syn. Rhynchostegium tenellum [Lorentz 1867:53]; Hypnum tenellum Dicks.,1801; Rhynchostegiella algiriana (Brid.) Broth.[ Brotherus 1925: 377].

$\mathrm{S}$. 
168. Rhynchostegium riparioides (Hedw.) Cardot, in Tourret, Bull. Soc. Bot. France 60: 231.1913

Syn. Hypnum rusciforme Neck. [Decaisne 1834, cf. Hart 1891:171]; Rhynchostegium rusciforme [Lorentz 1867:53]; Hypnum ruscifolium Neck. [Hart 1891:170]; Platyhypnidium rusciforme ( Neck.) Fleisch. [Brotherus 1925:347].

[As Brachythecium rivulare by Imam \& Ghabbour 1972: 519; cf. Vana 1988; ElSaadawi \& Badawi 1977: 184; Abou Salama 1985: 126 and Frey \& Kürchner 1991: 151]

S.

\section{Excluded taxa:}

\section{a) Misidentified taxa:}

Triquetrella papillata (Hook. \& Wilson.) Broth. Nf (App.1; 7, 25)

b) Taxa collected from beyond Egyptian boundaries:

× Hyophila laxitexta Froelich, nomen nudum, GE, Sudanese territory (App.1; 38). Hymenostomum laxirete (Broth.) Broth., GE, Sudanese territory, misidentified (App.1; 22).

\section{c) Greenhouse and garden taxa:}

Amblystegium varium (Hedw.) Lindb., Mma (App.1; 22, 38, 57).

Bryum atrovirnes Vill. ex Brid., Cai, Nv (App.1; 38), Cai (App.1; 22).

\# B. sauteri Bruch, Schimp.\& W. Gumbel, Cai (App.1; 63).

\# Hymenostomum laxirete (Broth.) Broth., Cai misidentified (App.1; 23).

Oxystegous sp., Cai, misidentified (App.1;23)

Pleuridium subulatum (Hedw.) Rabenh., Cai (App.1; 20); Mma (App.1; 22) .

Pohlia sp., Cai (App.1; 20).

Splachnobryum sp., Cai (App.1; 17).

œ\#Gmnostomiella laevis Fröhlich, nomen nudum, Cai (App.1; 22, 38).

\section{d) Nomina nuda:}

\# Barbula sickenbergeri Müll. Hal.,Mma (App.1; 60).

\# Bartramia nilotica Müll. Hal. (=a Philonotula nilotica), Cai (App.1; 60)

\# Bryum glauculum Müll. Hal. ex Sickenb., Nv, Nn ( App.1, 60)

\# Entosthodon schweinfurthii Müll. Hal., Cai (App.1; 60)

a\#Hypnum klunzingeri Müll. Hal., R (App.1; 39)

In addition to the above 16 taxa Sickenberger (1901), reported a\#Barbula muralis var. obovata Schimp. from Mariout (Mma, App. 1; 60). This moss is either a nomen nodum or the author ment to say var. obcordata which is a synonym of Toutula muralis var. obcordata (cf. also Ros et al. 1999). 
W. El-Saadawi, A. Badawi, H.M. Shabbara, U.Y. Abou Salama \& M.S.M. Refai

\section{Acknowledgements}

The authors are grateful to Prof. RNDr. J Vana of Charles University, Praha, Czech Republic for making his valuable unpublished report, on the bryophytes of Sinai available to us, and to Prof. Dr. M. N. El-Hadidi for providing facilities in CAI. We thank also Mr. Khaled Mahmoud, of Manchester Univ. who kindly provided us with photocopies of requested papers.

\section{Appendix 1: works dealing entirely or partly with Egyptian mosses:}

1- Abou Salama, U.Y. 1985. Morphological studies on some Egyptian bryophytes in Southern Sinai. M.Sc. Thesis. Botany Department. Faculty of Science. Ain Shams University. Cairo.

2- Abou Salama, U.Y. 1991. Studies on the bryoflora of Egypt (Southern Sinai) with special reference to genus Bryum. Ph.D. Thesis. Botany Department. Faculty of Science. Ain Shams University. Cairo.

3- Abou Salama, U.Y. 1999. Mosses of the Egyptian conservation Areas: 1- Gebel Elba Protectorate. Phytomorphology (In press).

4- Amann, J.J. 1895. Une Mousse Nouvelle d' Egypte. Bull. Herb. Boissier, 3 (8): 442444.

5- Amann, J.J. 1922. Fissidens Mnevidis Amann. Revue Bryologique 49: 51.

6- Badawi, A., El-Saadawi W. \& Refai M. 1987a. The Moss flora of Egypt. 2- The Nile Delta. Ain Shams Science Bulletin 26B: 183- 230.

7- Badawi, A., El-Saadawi W. \& Shabbara H. 1987b. On the mosses of El Fayum region, Egypt. Ibid., 26B: 357-3 72.

8- Bilewsky, F. 1965. Moss flora of Israel. Nova Hedwigia 9: 335-434.

9- Bilewsky, F. 1974. Some notes on the distribution of mosses in Israel and Palestine. Rev. Bryol. Lichenol 40: 245-261.

10-Boulos, L. 1966. Flora of the Nile Region in Egyptian Nubia. Feddes Repertorium 73: 184- 215.

11- Brotherus, V.F. 1924-1925. In Engler, A. \& Prantle K. Die Natürlichen Pflanzenfamilien. 10, 1924; 11, 1925. Duncher and Humblot, Berlin.

12- Bruggeman-Nannenga, M.A. 1982. The section Pachylomidium (genus Fissidens) III. The F . crassipes- subcomplex (F. bryoides- complex), F. sublineaefolius (Pot. Varde) Brugg.-Nann. and $\boldsymbol{F}$. fluitans (Pot. Varde) Brugg.-Nann. Proceedings of the Koninklijke Nederlandsee Akademie van Wetenschappen, series C 85: 59- 104.

13- Crundwell A.C.\& Nyholm E. 1974. Funaria muhlenbergii and related European species. Lindbergia 2: 222 - 229.

14-Decaisne, J. 1834. Florula Sinaica. Enumeration des plantes recuellees par M. Bove dans les deux Arabies, la Palestine, la Syrie et l' Egypt. Ann. Sci. Nat. 2, ser.2: 518.

15- Delgadillo, C.M. 1975. Taxonomic revision of Aloina, Aloinella and Crossidium (Musci). The Bryologist 78: 243- 303.

16-Delile, R. 1813. Flora Agyptica Illustratio Description de L'Egypte. Histoire Naturelle Paris 2. 289. 
17-El-Awadi, H. 1985. Morphological studies on some Egyptian bryophytes in Cairo region. M.Sc. Thesis. Botany Department. Faculty of Science. Ain Shams University. Cairo.

18- El-Saadawi, W.E. 1972. Illustrated description of Micropoma niloticum (R. Delile) Lindb. Proceeding of the Egyptian Acad. of Sci. 25: 217- 219.

19-El-Saadawi, W. 1995. Bryophytes and Pteridophytes. Publications of National Biodiversity unit 3: 59-60. United Nations Environment Programme (UNEP).

20-El-Saadawi, W.\& Abou El- Kheir, W.S. 1973. On some Egyptian mosses and the algal flora in their habitats. Ibid., 26: 125- 136.

21- El-Saadawi, W., Abou El-Kheir W.S.\& Darwish M.H. 1986a. Notes on Egyptian mosses with one new record. Lindbergia 12: 129- 131.

22- El-Saadawi, W.\& Badawi A. 1977. The moss flora of Egypt 1-Order Pottiales. Public. Cairo University Herbarium 7-8: 181- 206.

23- El-Saadawi, W., Badawi A. \& El-Awadi H.1987a. The moss flora of Egypt 3. The Cairo Region. Ain Shams Science Bulletin 26 B: 295- 320.

24- El-Saadawi, W., Badawi A.\& Refai M.S. 1986b. Mosses of the Nile Delta. Lindbergia 12: 106- 110 .

25- El-Saadawi, W., Badawi A.\& Shabbara H. 1987b.The moss flora of Egypt. IV. ElFayum Province. Ain Shams Sci. Bull. 26B: 321- 356.

26- Frahm, J.P. 1982. Personal communication

27- Frey W.\& Kürschner H.1988a. Bryophytes of the Arabian Peninsula and Socotra. Floristics, Phytogeography and definition of the Xerothermic Pangaean element. Studies in Arabian bryophytes 12. Nova Hedwigia 46: 37-120.

28- Frey W. \& Kürschner H.1988b. Re-evaluation of Crossidium geheebii ( Broth.) Broth. (Pottiaceae) from Sinai, a Xerothermic Pangaean element. Journal of Bryology 15: 123- 126.

29- Frey, W. \& Kürschner, H. 1991. Conspectus Bryophytorum Orientalum et Arabicorum, an annotated catalogue of the bryophytes of southwest Asia. J. Cramer. Berlin. Stuttgart.

•30- Gallego M.T., Cano M.J., Ros R.M.\& Guerra J. 1999. The genus Aloina ( Pottiaceae, Musci) in the Mediterranean region and neighbouring areas. Nova Hedwigia 69 (1-2): 173- 194.

31- Geheeb A. 1903. Bryophyta.- In Kneucker A., Botanische Ausbeute einer Reise durch die Sinaihalbinsel vom 27. Marz bis 13. April 1902.- Allg. Bot. Z. Syst. 1903: 185189, 203-204

32- Geheeb A. 1904. Bryophyta.- In Kneucker A., Botanische Ausbeute einer Reise durch die Sinaihalbinsel vom 27. Marz bis 13. April 1902.-Ibid., 1904: 4-5

33- Hart, H.C. 1891. Some Account of the Fauna and Flora of Sinai, Petra, and Wady Arabah. London.

34- Hedwig, I. 1816. Species Muscorum Frondosorum suppl. Vol. II.

35- Herranstadt, L., Heyn C.C., Ben- Sasson R. \& Crosby M.R.1982. New records of mosses from Israel. The Bryologist 85: 214-217.

36- Hooker J.D. \& Hart H.C. 1861-1885. Syrian Botany. London.

- This publication appeared shortly before the present paper went to press. 
37- Hume, W.F. 1906. The topography and geology of the peninsula of Sinai ( southeastern portion). Cairo.

38- Imam, M. \& Ghabbour, S.I. 1972. A contribution to the moss flora of Egypt. Bot. Notiser 125 (4): 518-522.

39- Klunzinger, C.B. 1878. Die Vegetation der egyptisch-arabischen Wüste bei Koseir. Zeitschrift der Gesellschaft für Erdkunde zu Berlin, XIII, Berlin.

40- Lashin, G.M. 1990. Studies on the bryoflora of Suez Canal Region. M. Sc. Thesis. Botany Department. Faculty of Science. Zagazig University. Zagazig.

41- Lindberg, S.O. 1864. Uppställning af familjen Funariaceae. Öfvers af K. Vet-Akad. Förh. 10: 589-608.

42- Lorentz, P.G. 1867. .ber die Moose, die Herr Ehrenberg in den Jahren 1820 - 1826 in Ägypten, der Sinai-halbinsel und Syrien gesammelt hat. Phys. Math. Abh. K. Akad. Wiss. Berlin, 1-57.

43- Mansi, M.A. 1988. Studies on bryoflora of Southern Sinai. M. Sc. Thesis. Botany Department. Faculty of Science. Suez Canal University. Ismaelia.

44- Müller, C. 1849, 1851. Synopsis muscorum frondosorum. 2. Bd.- Berlin. (cf. Vana, 1988)

45- Müller, C. 1858. Additamenta nova ad Synopsin muscorum. Botanische Zeitung. 16. (24): 153-156.

46- Müller, K. 1874. Die Moose der Rohlfs'schen Expedition nach der Libyschen Wüste. Flora. Jahrg. 57(31): 481-485. Regensburg.

47- Ochi, H. 1972. A revision of African Bryoideae, Musci (1st. part). The journal of Faculty of Education Tottori Univ. 24 (1), Koyamacho, Tottori, Japan.

48- Ochyra, R. 1995. The taxonomic status of Amblystegium burnatii (Amblystegiaceae), an endemic Egyptian moss Fragm. Flor. Geobot. 40 (1): 201- 202.

49- Podpera, J. 1953. Bryum generis monographiae prodromus.1. Species Eurasiae septentrionalis. Pars 8. Systematica.- Prace Morav.-Slez. Akad. Ved Prir. 25(2): 53- 120.

50- Refai, M. S. 1985. Studies on the Bryoflora of The Nile Delta. M. Sc. Thesis. Botany Department. Faculty of Science. Ain Shams University. Cairo.

51- Refai, M.S. 1991. Studies on the bryoflora of Egypt (The Nile Valley) with special reference to genus Barbula. Ph.D. Thesis, Botany Department, Faculty of Science, Ain Shams University, Cairo.

52- Renauld, F. \& Cardot, J. 1894. Mosses Nouvelles de L' Herbier Boissier. Bull. Herb. Boissier 2 (1).

-53- Ros, R., Cano, M. \& Guerra, J.1999. Bryophyte checklist of northern Africa. Journal of Bryology 21: 207- 244.

54- Schimper, W. 1855. Cited from Müller 1858.

55- Schimper, W. 1876. Synopsis muscorum europaeorum vol.2. Stuttgart. (cf. Vana 1988)

56- Shabbara, H.M. 1986. Morphological Studies on the Bryophytes of El- Fayum. M. Sc. Thesis. Botany Department. Faculty of Science. Ain Shams University. Cairo.

\footnotetext{
- This publication appeared shortly before the present paper went to press.
} 
57- Shabbara, H.M. 1990. Studies on the bryoflora of the Mediterranean Coast of Egypt. Ph.D. Thesis. Botany Department. Faculty of Science. Ain Shams University. Cairo.

58- Shabbara, H.M. 1997. The first record of a fruiting Gymnostomum Nees et Hornsch. (Musci) in Egypt. Egypt. J. Bot.37 (1): 75- 84.

59- Shabbara, H.M. 1999. Three new records of Funariaceae from Egypt. Journal of Bryology 21: 201- 205.

60- Sickenberger, E. 1901. Contribution a la Flora d'Egypte. Memoire de l'Institute d'Egypte 4: 1- 167.

61- Smith, A.J.E. 1978. The moss flora of Britain and Ireland. Cambridge Univ. Press.

62- Syed, H. 1973. A taxonomic study of Bryum capillare Hedw. and related species. Journal of Bryology 7: 265- 326.

63- Townsend, C.C. 1996. Bryum sauteri B.,S. and G. in Egypt. Journal of Bryology 19: 348-349.

64- Vana, J. 1988. Unpublished report on the Bryophytes of Sinai.

65- Warnstorf, C. 1911. Verzeichnis der von M. Fleischer 1908 während der Monate April und Mai in Südfrankreich und Spanien beobachteten Laub-, leber- und Torfmoose. Hedwigia 50: 189- 203.

66- Whitehouse, H.L.K. \& Crundwell A.C. 1991. Gymnostomum calcareum Nees \& Hornsch. and allied plants in Europe, North Africa and the Middle East. Journal of Bryology 16: 561- 579.

67- Youssef, S.G.M. 1987. Morphological studies on the bryophytes of El-Sharkia Governorate. M.Sc. Thesis. Botany Department. Faculty of Science. Zagazig University. Benha, Egypt.

\section{References}

Anderson, L.E., Crum H.A.\& Buck W.R. 1990. List of mosses of North America, North of Mexico. The Bryologist 93:448-500.

Brummitt, R.K.\& Powell C.E. 1992. Authors of Plants Names. Royal Botanic Gardens Kew.

Corley, M.F.V., Crundwell A.C., Düll R., Hill M.O.\& Smith A.J.E. 1981. Mosses of Europe and the Azores; an annotated list of species, with synonyms from the recent literature. Journal of Bryology 11:609-689.

Crosby, M.R. 1977. Index Muscorum supplementum: 1974-1975. Taxon 26:285-307.

Crosby, M.R. 1979. Index Muscorum supplementum: 1976 - 1977. Ibid., 28 (1,2,3):237264.

Crosby, M.R.\& Bauer C.R. 1981. Index Muscorum supplementum : 1978 - 1979. Ibid., 30:667-693.

Crosby, M.R.\& Bauer C.R.1983. Index Muscorum supplementum :1980 - 1981. Ibid., 32 (4): 670-691.

Crosby, M.R., Magill R.E.\& Bauer C.R. 1992. Index of Mosses 1963- 1989. Monographs in systematic Botany from the Missouri Botanical Garden 42:1-646.

Crosby, M.R.\& Magill R.E. 1994.Index of Mosses 1990- 1992.Ibid.,50: 1- 87.

El- Hadidi, M.N.\& Fayed, A.A. 1994/95. Materials for Excursion Flora of Egypt (EFE) Täckholmia 15: 1-233. 
Herranstadt, L., Heyn C.C.\& Crosby M.R. 1991. A checklist of the mosses of Israel. The Bryologist 94(2): 168-178.

Sayre, G., Bonner C.E.B.\& Culberson W. L. 1964. The authorities for the epithets of mosses, hepatics and lichens.- The Bryologist 67: 113- 135.

Täckholm, V. 1974. Student's Flora of Egypt. Cairo Univ.

Wijk, R., Margadant W.D. \& Florschiitz P.A. 1959-69. Index Muscorum, 1-5, Regnum vegetabile $\mathbf{1 7 , 2 6 , 3 3 , 4 8} \& 65$.

Zander, R.H.1993. Genera of the Pottiaceae: Mosses of Harsh Environments. Bulletin of the Buffalo Society of Natural Sciences .32. Buffalo, N.Y. 